\title{
Snake-Like Units Using Flexible Backbones and Actuation Redundancy for Enhanced Miniaturization
}

\author{
Nabil Simaan* \\ Department of Mechanical Engineering \\ Columbia University \\ New York, New York 10027 \\ ns2236@columbia.edu
}

\begin{abstract}
This paper reports some recent analysis and modeling results obtained while developing a tele-robotic system for minimally invasive surgery of the throat. One of the main enabling components of this system is a Distal Dexterity Unit that implements a novel design using a flexible multi-backbone snake-like unit with actuation redundancy and push-pull actuation. The design of this snake-like unit is compared to other alternative designs that use a single flexible-backbone and wire-actuation. A unified kinematic and virtual work model is used to perform this comparison between a multi-backbone snake like unit with an equaldiameter snake-like unit using a single flexible backbone and wire actuation. The comparison is presented for several actuation redundancy resolutions that minimize the load on the flexible backbones. The results show that the multibackbone design is superior to the alternative wire-actuated designs using a single flexible backbone. The advantages manifest in smaller required actuation forces on the backbones and, as a result, a reduced risk of buckling of the backbones and enhanced potential downsize scalability.
\end{abstract}

Index Terms - Flexible backbone robot, snake robots, surgical assistant, master-slave mode, actuation redundancy.

\section{INTRODUCTION}

In our previous papers [1, 2], we reported our efforts to develop a high-dexterity robotic system for Minimally Invasive Surgery (MIS) of the throat. The primary enabling mechanical component of this system is a novel Distal Dexterity Unit (DDU) that overcomes the constraint to the traditional four Degrees-of-Freedom (DoF) associated with standard rigid tools used in MIS. This DDU enables complex tissue manipulation tasks in confined spaces that are otherwise very difficult to perform. The DDU is a hybrid five-DoF robot composed from a parallel manipulation unit and a Snake-Like Unit (SLU). This SLU features a novel design that uses several flexible backbones, actuation redundancy, and push-pull actuation. This paper focuses on the advantages of this SLU over wire-actuated designs.

Snake robots have been widely investigated for various applications that benefit from their high dexterity and obstacle-avoidance capabilities (see [3], [4] for a historical perspective). Due to their inherent large number of DoF, solving the inverse kinematics efficiently was one of the main theoretical problems that precluded their use. Chirikjian [5] and Zanganeh [6] addressed this problem by using variational calculus methods. Early articulated

\footnotetext{
* This research was conducted while Nabil Simaan was at Johns Hopkins University ERC-CISST (www.cisst.org), Baltimore, Maryland 21218.
}

designs of snake robots were very cumbersome to construct, required many mechanical joints and; hence, suffered from serious mechanical backlash problems [4]. To overcome these problems, several works by Walker and Gravagne, [7-9], addressed the kinematics and control of flexible continuous backbone designs that inspired this work. While all these works used designs based on single flexible backbone actuated by wires, we use in our design several flexible backbones actuated in push-pull modes.

Applications of snake-like robots for surgical tool manipulation are gaining increased attention from the research community. For example Dario et al. [10] presented a 1 DoF planar SMA actuated bending snake-like device for knee arthroscopy. Recently, Reynaerts et al. [11] presented a two DoF $5 \mathrm{~mm}$ diameter wire-actuated snakelike tool using super-elastic NiTi tube with flexure joints. Guthart and Salisbury [12] developed a snake-like robot with a discrete backbone and wire actuation. More recent works also presented miniaturized linkage designs for snake-like robots [13], wire actuated robots [14], and snake-like units with miniature gear-motors embedded inside the links [15].

In this paper we compare the proposed design of our SLU with other alternative wire-actuated designs such as the single-flexible backbone design. We focus on the required actuation forces and the compression loads on the backbones. Our main premise is that these compression loads are a major hindrance for downsize scalability of SLU's due to the buckling of their backbones. We will show in this paper that this problem is exacerbated in wireactuated designs that rely on a single flexible backbone.

\section{THE BENDING SNAKE-LIKE UNIT}

Figure 1 presents the DDU of our system. It is composed from a SLU and a 3 DoF parallel manipulation unit. The SLU is a two DoF mechanism that enables the surgeon to orient surgical tools in any direction in 3D. The parallel manipulation unit is for high-accuracy motions and for attaching different surgical tools to the tip of the SLU.

The SLU is composed from a base disk, several spacer disks, an end disk and four flexible super-elastic hollow tubes that we call backbones. One out of these four backbones is rigidly attached to all disks, Fig. 1. This backbone is called the primary backbone and it is located at the center of the SLU between three other secondary backbones that are radially displaced around it and equidistant from each other. All secondary backbones are connected only to the end disk. The spacer disks maintain fixed radial distance between the secondary backbones and the primary backbone. By actively changing the lengths of 
two out of the three secondary backbones the end disk can be manipulated in 2 DoF to be oriented in any required direction. In this work we use three secondary backbones to provide actuation redundancy for optimizing the distribution of the loads between the secondary backbones and the primary backbone.

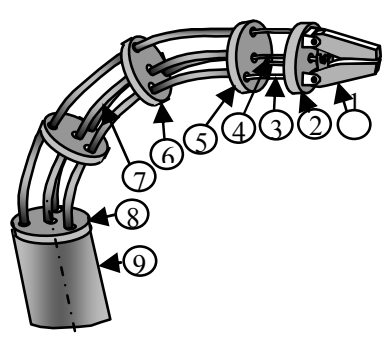

\begin{tabular}{|l|l|}
\hline 1 & gripper \\
\hline 2 & moving platform \\
\hline 3 & parallel stage wires \\
\hline 4 & gripper wire \\
\hline 5 & end disk \\
\hline 6 & spacer disk \\
\hline 7 & $\begin{array}{l}\text { central backbone } \\
\text { tube }\end{array}$ \\
\hline 8 & base disk \\
\hline 9 & DDU holder \\
\hline
\end{tabular}

Fig. 1 The Distal Dexterity Unit (DDU) composed from a multibackbone snake-like robot equipped with a parallel tip.

The advantages of the design in Fig. 1 were presented in $[1,2]$. These advantages include a high number of DoF, design simplicity, and backlash elimination due to the use of flexible backbones that remove the dependency on highprecision miniature mechanical joints. All the backbones of the SLU are hollow tubes that can be used for secondary purposes. This provides the SLU with multi-functionality in the surgical arena for applications such as surgical tool manipulation, aspiration, drug delivery, controllable light source, and visualization. However, these advantages come with a price of complicated kinematics and design.

In this paper, we focus on the pros of using push-pull actuation through the secondary backbones. The first obvious alternative to this design is replacing the secondary backbones of Fig. 1 with three wires. In sections VI, VII, we will compare this alternative design to our design in terms of resultant compressive forces on the backbones, and the corresponding implications on downsize scalability.

The first obvious advantage of the design of Fig. 1 is illustrated in Fig. 2. The figure shows that the minimal number of wires required to bend the central backbone in any direction is three while in the case of push-pull actuation it is two. In wire actuation, the wires apply on the central backbone a resultant bending moment $\mathbf{m}_{\mathrm{r}}$ that is a combination of their uni-directional actuation bending moments $\mathbf{m}_{\mathrm{i}} \mathrm{i}=1,2,3$. Since the actuation moments are uni-directional then a pair of wires spans only a convex cone of possible resultant moments. To fully cover the plane, three convex cones are required. Hence, the minimal number of actuation wires is three. If push-pull actuation is used, Fig. 2-b, then any resultant bending moment can be spanned by a linear combination of two actuation moments $\mathbf{m}_{1}$ and $\mathbf{m}_{2}$; hence, two actuated backbones are sufficient.

Figure 3 shows an early model of the SLU of Fig. 1. The figure shows the central backbone before its failure. This unit was bent by pulling on one backbone only. The primary backbone of this unit buckled when the actuated

\footnotetext{
- A convex cone of vectors $\mathbf{a}$ and $\mathbf{b}$ is defined as all their positive linear combinations: $\mathbf{c}=\{\alpha \mathbf{a}+\beta \mathbf{b} \mid \alpha, \beta \geq 0\}$.
}

secondary backbone (backbone no. 1 in Fig. 3) was pulled on to increase the bending angle of this unit. The improved design of Fig. 1 uses three secondary backbones that are all actuated in push-pull mode to prevent this failure.

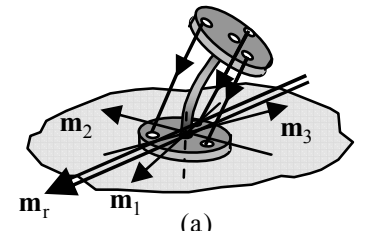

(a)

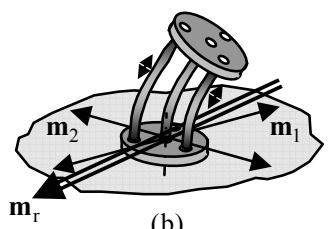

(b)
Fig. 2 The resultant bending moment, $\mathbf{m}_{\mathrm{r}}$, as a linear combination of actuation moments. Wire actuation (a) push-pull actuation (b).

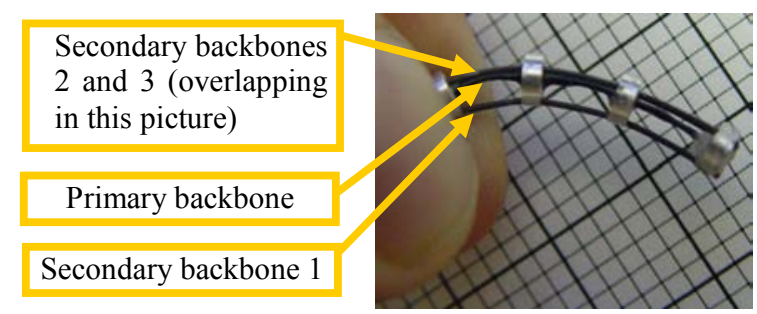

Fig. 3 A picture of an early SLU model an instance before its primary backbone buckled. It was actuated by pulling on one secondary backbone only.

The first prototype of the SLU was presented in [1]. Figure 4 shows an improved $4.2 \mathrm{~mm}$ diameter version of this SLU that is currently used for initial experiments. It is designed to apply $1 \mathrm{~N}$ at its tip in any direction. In fig. 4 it is manually actuated by using two backbones in push-pull mode, it is capable of applying more than 1 Newton at its tip and bending more than $70^{\circ}$ in any direction [2].
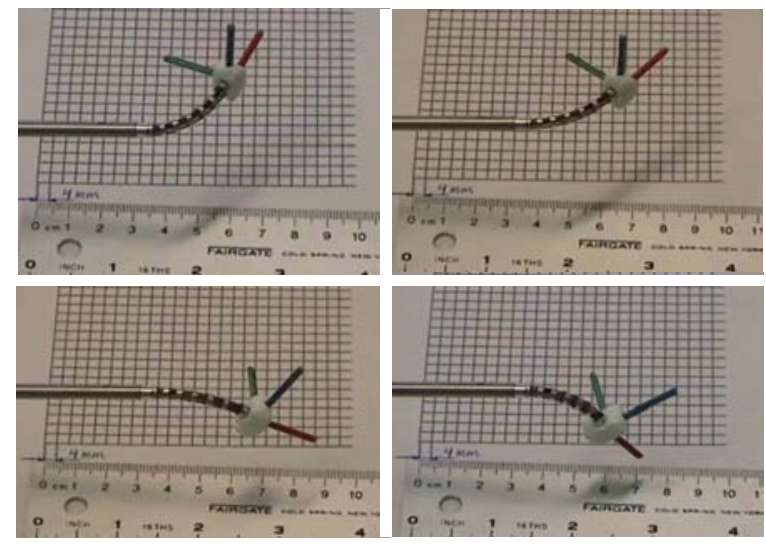

Fig. 4 The prototype of the SLU performing spatial motions

\section{KINEMATIC MODELLING}

The outline for the direct kinematics and the instantaneous kinematics of the SLU was presented in [1]. In this paper, we assume that the SLU is installed on a rotation stage that rotates its base disk. We also include a gripper that is affixed to the end disk of the snake and we define additional transformations accordingly.

Figure 5 shows the SLU with only the primary and one secondary backbone illustrated. Three coordinate systems were defined in [1]: the Base Disk coordinate System 
(BDS) $\left\{\hat{\mathbf{x}}_{\mathrm{b}}, \hat{\mathbf{y}}_{\mathrm{b}}, \hat{\mathbf{z}}_{\mathrm{b}}\right\}$, the Snake Plane coordinate System (SPS) $\left\{\hat{\mathbf{x}}_{1}, \hat{\mathbf{y}}_{1}, \hat{\mathbf{z}}_{1}\right\}$, the End Disk coordinate System (EDS) $\left\{\hat{\mathbf{x}}_{\mathrm{e}}, \hat{\mathbf{y}}_{\mathrm{e}}, \hat{\mathbf{z}}_{\mathrm{e}}\right\}$. In addition, we include here the gripper coordinate system $\left\{\hat{\mathbf{x}}_{\mathrm{g}}, \hat{\mathbf{y}}_{\mathrm{g}}, \hat{\mathbf{z}}_{\mathrm{g}}\right\}$ and the World Coordinate System (WCS) $\left\{\hat{\mathbf{x}}_{\mathrm{w}}, \hat{\mathbf{y}}_{\mathrm{W}}, \hat{\mathbf{z}}_{\mathrm{w}}\right\}$ to facilitate the description of suturing tasks in confined spaces in section IV.

The gripper coordinate system is attached to an imaginary gripper affixed to the end disk. The three secondary backbones are equidistant from each other and from the central backbone. They are numbered as in Fig. 5 according to the right-hand rule about $\hat{\mathbf{z}}_{\mathrm{b}}$. Without loss of generality, we assume that the base disk lies at the origin of WCS and that $\hat{\mathbf{z}}_{\mathrm{w}}$ is normal to it. Unit vector $\hat{\mathbf{x}}_{\mathrm{b}}$ is defined such that its points from the center of the base disk to the first secondary backbone. SPS is defined such that the plane in which the snake-like unit bends is defined by unit vectors $\hat{\mathbf{x}}_{1}$ and $\hat{\mathbf{z}}_{1}$. EDS is obtained from SPS by a simple rotation about $\hat{\mathbf{y}}_{1}$ such that $\hat{\mathbf{z}}_{1}$ becomes the backbone tangent at the end disk. The gripper coordinate system is defined like BDS, but it is physically attached to the end disk. As such, $\hat{\mathbf{x}}_{\mathrm{g}}$ points from the center of the end disk to the first secondary backbone and $\hat{\mathbf{z}}_{\mathrm{g}}$ is normal to the end disk.

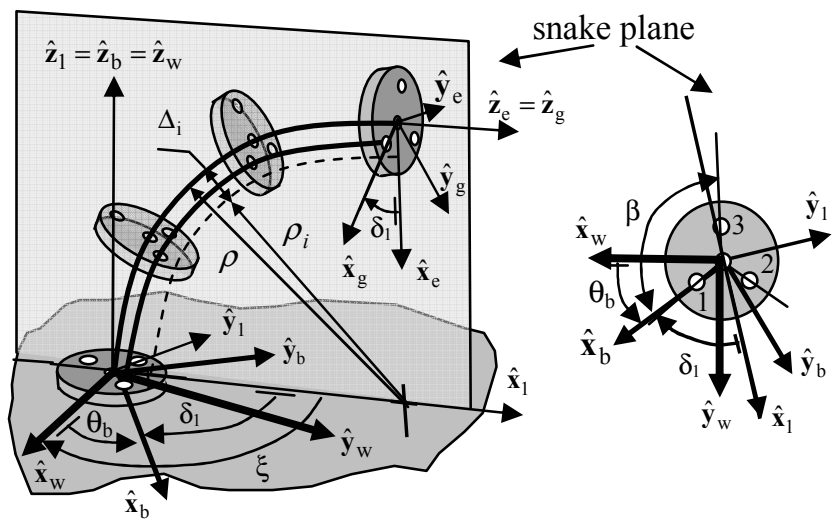

Fig. 5 Kinematic nomenclature for the SLU. The SLU with a central and a secondary backbone shown (left). Top view of the base disk (right).

The following symbols are used:

- index of the secondary backbones $i=1,2,3$.

$\mathrm{s} \quad$ - arc-length parameter of the primary backbone. $\mathrm{s}=0$ at the base disk and $\mathrm{s}=\mathrm{L}$ at the end disk.

$\mathrm{L}_{\mathrm{i}} \quad$ - length of the $\mathrm{i}^{\text {th }}$ secondary backbone measured from the base to the end disk.

$\mathrm{r} \quad$ - radius of the base, spacer, and end disks.

$\rho(\mathrm{s})$ - radius of curvature defined as $|\mathrm{ds} / \mathrm{d} \theta(\mathrm{s})|$.

$\theta(s)$ - the angle of the primary backbone tangent in the $\hat{\mathbf{x}}_{1} \hat{\mathbf{z}}_{1}$ plane. $\theta(\mathrm{s}=\mathrm{L})$ and $\theta(\mathrm{s}=0)$ are designated by $\theta_{\mathrm{L}}$ and $\theta_{0}$, respectively.

$\theta_{\mathrm{b}} \quad$ - is the angle of rotation of the base disk about $\hat{\mathbf{z}}_{\mathrm{b}}$.

$$
\theta_{\mathrm{b}}=\operatorname{Atan} 2\left(\hat{\mathbf{x}}_{\mathrm{b}}{ }^{\mathrm{t}} \hat{\mathbf{y}}_{\mathrm{w}} / \hat{\mathbf{x}}_{\mathrm{b}}{ }^{\mathrm{t}} \hat{\mathbf{x}}_{\mathrm{w}}\right) \text {. }
$$

$\beta$ - division angle $(\beta=2 \pi / n$ where $n$ is the number of secondary backbones).

$\delta_{i} \quad$ - the right-handed rotation angle from $\hat{\mathbf{x}}_{1}$ about $\hat{\mathbf{z}}_{1}$ to a line passing through the primary backbone and the $i$ 'th secondary backbone at $\mathrm{s}=0$. $\delta_{i}=\delta+(i-1) \beta, \quad i=1,2,3$.

$\delta$ - the angle of the snake plane. It is defined as $\delta=\delta_{1}$.

$\Delta_{\mathrm{i}}$ - the offset from the primary backbone to the projection of the i'th secondary backbone on the snake plane.

$\xi \quad$ - the right-handed rotation from $\hat{\mathbf{x}}_{1}$ to $\hat{\mathbf{x}}_{\mathrm{w}}$ about $\hat{\mathbf{z}}_{1}$.

$\dot{\mathrm{x}} \quad$ - time derivative of variable $\mathrm{x}$.

$\mathbf{J}_{\mathrm{xy}}-$ Jacobian matrix such that $\dot{\mathbf{x}}=\mathbf{J} \dot{\mathbf{y}}$.

$\mathrm{E}_{\mathrm{yp}}, \mathrm{E}_{\mathrm{ys}}-$ Young's modulus for the primary and secondary backbones, respectively.

$I_{p}, I_{s}$ - the cross-sectional moments of inertia. $I_{p}$ is used for primary and $\mathrm{I}_{\mathrm{s}}$ for secondary backbones.

As in [1] the analysis assumes a quasi-static motion. The direct kinematics of the snake is represented in a 2dimensional space such that the position and orientation (pose) of the end disk relative to the base disk is characterized by two angles $\theta_{\mathrm{L}}$ and $\delta$. The length of the i'th backbone depends on the angle of the end disk relative to the base disk, but not on the shape of the backbone, [1], [7].

$$
\mathrm{L}_{\mathrm{i}}=\mathrm{L}+\Delta_{\mathrm{i}}\left(\theta_{\mathrm{L}}-\theta_{0}\right) \quad \theta_{\mathrm{L}} \in\left[0, \theta_{0}\right]
$$

For given $\mathrm{L}_{1}$ and $\mathrm{L}_{2}$, the angle $\theta_{\mathrm{L}}$ is found by:

$$
\theta_{\mathrm{L}}=\theta_{0}-\left(\mathrm{L}-\mathrm{L}_{\mathrm{i}}\right) / \Delta_{\mathrm{i}}
$$

where $\Delta_{\mathrm{i}} \equiv \mathrm{r} \cos \left(\delta_{\mathrm{i}}\right) \quad \mathrm{i}=1,2,3$. The angle of the snake plane $\delta$ is found by imposing a kinematic compatibility condition stating that the angle $\theta_{\mathrm{L}}$, as found from Eq. (1) for $\mathrm{i}=1$, and 2, is the same [1]. The two solutions in Eq. (3) represent a single configuration of the snake-line unit and correspond for two opposing directions of $\hat{\mathrm{x}}_{1}$ of Fig. 5 .

$$
\delta=\operatorname{Atan} 2(\mathrm{~A} / \mathrm{B}) \quad \text { or } \operatorname{Atan} 2(\mathrm{~A} / \mathrm{B})+\pi
$$

where $\mathrm{A}$ and $\mathrm{B}$ were defined in [1] as:

$$
A=\left[\left(L-L_{1}\right) \cos (\beta)-\left(L-L_{2}\right)\right], \quad B=\left[\left(L-L_{1}\right) \sin (\beta)\right](4)
$$

The length of the third secondary backbone, $\mathrm{L}_{3}$, can be found by using Eq. (2). The position, $\mathbf{p}_{\mathrm{L}}$, and the orientation, ${ }^{\mathrm{w}} \mathbf{R}_{\mathrm{g}}$, of the gripper are found by integrating along the tangent of the backbone and by a successive rotation sequence as in Eq. (5). The matrix ${ }^{\mathrm{w}} \mathbf{R}_{\mathrm{b}}$ is a rotation of $\theta_{\mathrm{b}}$ about $\hat{\mathbf{z}}_{\mathrm{w}},{ }^{\mathrm{b}} \mathbf{R}_{1}$ is a rotation matrix of $(-\delta)$ about $\hat{\mathbf{z}}_{\mathrm{b}},{ }^{1} \mathbf{R}_{\mathrm{e}}$ is a rotation matrix of $\left(\theta_{0}-\theta_{\mathrm{L}}\right)$ about $\hat{\mathbf{y}}_{1}$, ${ }^{\mathrm{e}} \mathbf{R}_{\mathrm{g}}$ is a rotation matrix of $\delta$ about $\hat{\mathbf{z}}_{\mathrm{e}}$. 


$$
\begin{aligned}
\mathbf{p}_{\mathrm{L}}={ }^{\mathrm{w}} \mathbf{R}_{1}\left[\int_{0}^{\mathrm{L}} \cos (\theta(\mathrm{s})) \mathrm{ds}, 0, \int_{0}^{\mathrm{L}} \sin (\theta(\mathrm{s})) \mathrm{ds}\right]^{\mathrm{t}}, \\
{ }^{\mathrm{w}} \mathbf{R}_{\mathrm{g}}={ }^{\mathrm{w}} \mathbf{R}_{\mathrm{b}}{ }^{\mathrm{b}} \mathbf{R}_{1}{ }^{1} \mathbf{R}_{\mathrm{e}}{ }^{\mathrm{e}} \mathbf{R}_{\mathrm{g}}
\end{aligned}
$$

These results depend on a shape function, $\theta(\mathrm{s})$, that minimizes the potential energy of the system. For an ideal light-weight and unloaded SLU, $\theta(\mathrm{s})$ will be a circular section of length L [7]. Otherwise, numerical or direct variational methods can be used to approximate $\theta(\mathrm{s})$.

\section{SUTURING IN CONFINED SPACES}

The previous section can easily be used to enable suturing in confined spaces without resorting to using the inverse Jacobian relationship as often done for serial manipulators. The chosen mode-of-operation is called "rotation about the primary backbone" mode. In this operation mode, the SLU transforms rotation of its base disk to rotation of its end disk about its normal $\hat{\mathbf{z}}_{\mathrm{g}}$ while maintaining its end point fixed. If the rotation point is located at the center of a circular suturing needle, then suturing will be performed with minimal tissue tear while using the rotation of the snake-like unit about its backbone.

To achieve this goal, assume that the given start pose of the snake is characterized by $\delta=\delta_{\mathrm{S}}, \theta_{\mathrm{L}}=\theta_{\mathrm{Ls}}$ to which the corresponding SPS angle in WCS is $\xi_{\mathrm{s}}$. Assume also that the base disk rotates about $\hat{\mathbf{z}}_{\mathrm{w}}$ at an angular speed $\dot{\theta}_{\mathrm{b}}$. To maintain the snake end point fixed, the three secondary backbones are actuated such that the orientation of SPS in WCS remains fixed and the following constraint is fulfilled.

$$
\begin{array}{ll}
\xi_{\mathrm{s}}=\theta_{\mathrm{b}}-\delta=\text { constant } & \Rightarrow \dot{\delta}=\dot{\theta}_{\mathrm{b}} \\
\theta_{\mathrm{L}}=\theta_{\mathrm{Ls}}=\text { constant } & \Rightarrow \dot{\theta}_{\mathrm{L}}=0
\end{array}
$$

To fulfill this constraint, the inverse kinematics of the SLU is solved for $\delta(\mathrm{t})=\xi_{\mathrm{s}}+\theta_{\mathrm{b}}(\mathrm{t}), \theta_{\mathrm{L}}(\mathrm{t})=\theta_{\mathrm{Ls}}$.

Figure 6 demonstrates the rotation about the primary backbone on a large scale SLU installed on a rotation stage.

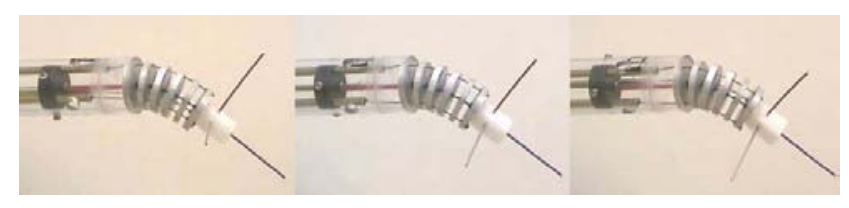

Fig. 6 A large snake-like unit rotating about its primary backbone

\section{VIRTUAL WORK MODEL}

The static analysis is based on a linearized virtual work model. The effects of the actuation forces and the gravitation potential energy on the total deformation energy of the backbones are neglected compared to the total deformation energy of the backbones. Under this assumption, the energy of the system of backbones is a function of their bending. The shape of the snake-like unit is characterized by $\theta(\mathrm{s})=\mathrm{f}\left(\theta_{\mathrm{L}}, \mathrm{s}\right)$ that minimizes the elastic energy of the backbones and by $\delta$. In a reverse direction, the elastic energy may be presented as a function of $\theta(s)=f\left(\theta_{L}, s\right)$ and $\delta$. Specifically for our SLU we write:

$$
\mathrm{E}_{\text {bending }}=\mathrm{E}\left(\theta_{\mathrm{L}}, \delta\right)=\left(\theta_{\mathrm{L}}-\theta_{0}\right)^{2}\left(\frac{\mathrm{E}_{\mathrm{yp}} \mathrm{I}_{\mathrm{p}}}{2 \mathrm{~L}}+\sum_{\mathrm{i}=1}^{3}\left(\frac{\mathrm{E}_{\mathrm{ys}} \mathrm{I}_{\mathrm{s}}}{2 \mathrm{~L}_{\mathrm{i}}}\right)\right)(7)
$$

Assume that an external wrench $\mathbf{w}_{e}=\left[\mathbf{f}_{e}{ }^{t}, \mathbf{m}_{e}{ }^{t}\right]^{t}$ acts on the end disk where $\mathbf{f}_{\mathrm{e}}$ indicates the force and $\mathbf{m}_{\mathrm{e}}$ the moment. This external wrench perturbs the posture (position and orientation) of the end disk by $\Delta \mathbf{x}$. To this pose perturbation there is a corresponding change $\Delta \mathbf{L} \equiv\left[\Delta \mathrm{L}_{1}, \Delta \mathrm{L}_{2}, \Delta \mathrm{L}_{3}\right]^{\mathrm{t}}$ in the lengths of the secondary backbones. The actuation forces that are required to act on the secondary backbones to maintain the equilibrium are $\boldsymbol{\tau} \equiv\left[\tau_{1}, \tau_{2}, \tau_{3}\right]^{\mathrm{t}}$. The change in the potential energy of the backbone system $\Delta \mathrm{E}$ that corresponds to $\Delta \mathbf{x}$ is given by:

$$
\mathbf{w}_{\mathrm{e}}{ }^{\mathrm{t}} \Delta \mathbf{x}+\boldsymbol{\tau}^{\mathrm{t}} \Delta \mathbf{L}-\Delta \mathrm{E}=0
$$

The virtual displacement of the SLU is characterized by $\Delta \psi=\left[\Delta \theta_{\mathrm{L}}, \Delta \delta\right]^{\mathrm{t}}$. The entities $\Delta \mathbf{x}$ and $\Delta \mathbf{L}$ are related to $\Delta \psi$ according to Eqs. (9).

$$
\Delta \mathbf{L}=\mathbf{J}_{\mathrm{L} \psi} \Delta \boldsymbol{\Delta} \quad \Delta \mathbf{x}=\mathbf{J}_{\mathrm{x} \psi} \Delta \psi
$$

where $\mathbf{J}_{\mathrm{x} \psi}$ is the instantaneous direct kinematics Jacobian outlined in [1] and $\mathbf{J}_{\mathrm{L} \psi}$ is found by taking the time derivative of Eq. (1) for $\mathrm{L}_{\mathrm{i}}, \mathrm{i}=1,2,3$.

Next, the virtual work principle is rewritten as in Eq. (10). The equilibrium condition requires the terms associated with each independent DoF to vanish. This results in a system of two equations in three unknowns $\boldsymbol{\tau} \equiv\left[\tau_{1}, \tau_{2}, \tau_{3}\right]^{\mathrm{t}}$. The matrix form of this system of equations is given in Eq. (11) where $\nabla \mathrm{E}$ represents the gradient of the elastic energy.

$$
\begin{gathered}
\mathbf{w}_{\mathrm{e}}{ }^{\mathrm{t}} \mathbf{J}_{\mathrm{x} \psi} \Delta \boldsymbol{\psi}+\boldsymbol{\tau}^{\mathrm{t}} \mathbf{J}_{\mathrm{L} \psi} \Delta \boldsymbol{\psi}-[\nabla \mathrm{E}]^{\mathrm{t}} \Delta \boldsymbol{\psi}=0 \\
\mathbf{J}_{\mathrm{L} \psi}{ }^{\mathrm{t}} \boldsymbol{\tau}=\nabla \mathrm{E}-\mathbf{J}_{\mathrm{x} \psi}{ }^{\mathrm{t}}{ }^{\mathbf{w}_{\mathrm{e}}}
\end{gathered}
$$

The resultant force on the primary backbone, $\tau_{p}$, is found from force equilibrium of the end disk in the $\hat{\mathbf{z}}_{\mathrm{e}}$ direction:

$$
\tau_{\mathrm{p}}=-\left(\sum_{\mathrm{i}=1}^{3} \tau_{\mathrm{i}}+\left(\mathbf{f}_{\mathrm{e}}{ }^{\mathrm{t}} \hat{\mathbf{z}}_{\mathrm{e}}\right)\right)
$$

By using $\sum_{\mathrm{i}=1}^{3} \tau_{\mathrm{i}}=\boldsymbol{\tau}^{\mathrm{t}} \mathbf{Z}_{\mathrm{e}}{ }^{\mathrm{t}} \hat{\mathbf{z}}_{\mathrm{e}}$ where $\mathbf{Z}_{\mathrm{e}}=\left[\begin{array}{lll}\hat{\mathbf{z}}_{\mathrm{e}} & \hat{\mathbf{z}}_{\mathrm{e}} & \hat{\mathbf{z}}_{\mathrm{e}}\end{array}\right]$, the condition $\tau_{\mathrm{p}}=0$ is given by:

$$
\mathbf{Z}_{\mathrm{e}} \boldsymbol{\tau}=-\mathbf{f}_{\mathrm{e}}
$$

\section{GENERALIZED REDUNDANCY RESOLUTIONS}

The SLU of Fig. 1 has one redundant secondary backbone. This backbone can be actuated to reduce the amount of force acting on the primary backbone, hence, reducing the risk of its buckling. This section presents a 
generalized redundancy resolution that achieves this goal. This is important to allow effective downsize scalability while preventing the thin backbones from buckling.

Eq. (11) is a system of two equations in three unknowns $\boldsymbol{\tau}$. Let $\mathbf{N}=\left(\mathbf{I}-\mathbf{A}^{+} \mathbf{A}\right)$ be the nullspace projector of $\mathbf{A}=\mathbf{J}_{\mathrm{L} \psi}{ }^{\mathrm{t}}$. Let $\mathbf{b}=\left(\nabla \mathrm{E}-\mathbf{J}_{\mathrm{x} \psi}{ }^{\mathrm{t}} \mathbf{w}_{\mathrm{e}}\right)$ be the generalized virtual work vector. The general solution of Eq. (11) is:

$$
\boldsymbol{\tau}=\mathbf{A}^{+} \mathbf{b}+\lambda \mathbf{N} \boldsymbol{\eta}
$$

where the $(+)$ superscript indicates the generalized inverse and $\boldsymbol{\eta} \in \mathfrak{R}^{3}$ is a vector of homogeneous actuation forces used to optimize the loads on the backbones and $\lambda$ is a scaling factor that scales the homogeneous solution $\mathbf{N} \boldsymbol{\eta}$

with respect to the particular solution $\mathbf{A}^{+} \mathbf{b}$. For SLU's that use secondary backbones in push-pull actuation $\lambda=1$.

Equation (14) can treat wire-actuated SLU's. In this case $\lambda$ is chosen such that all the wires are kept in tension and $I_{S}=0$ is used in Eq. (7). In our simulations we chose $\boldsymbol{\eta}=[1,1,1]^{\mathrm{t}}$, but any $\boldsymbol{\eta} \in \operatorname{col}(\mathbf{N})$ can be used [16]

Different redundancy resolutions use a different $\boldsymbol{\eta}$ in Eq. (14) based on the optimized criteria. Equation (15) defines a general objective function that accounts for the load on the primary backbone and the compressive loads on the secondary backbones.

$$
\underset{\boldsymbol{\eta}}{\operatorname{Min}} \mathrm{J}=\left(\mathbf{Z}_{\mathrm{e}} \boldsymbol{\tau}+\mathbf{f}_{\mathrm{e}}\right)^{\mathrm{t}} \mathbf{w}_{\mathrm{f}}\left(\mathbf{Z}_{\mathrm{e}} \boldsymbol{\tau}+\mathbf{f}_{\mathrm{e}}\right)+(\boldsymbol{\tau}-\tilde{\boldsymbol{\tau}})^{\mathrm{t}} \mathbf{w}_{\tau}(\boldsymbol{\tau}-\tilde{\boldsymbol{\tau}})_{(15)}
$$

$\tilde{\boldsymbol{\tau}}$ is the desired reference average actuation force. This function can be expressed in a matrix form:

$$
\mathrm{J}=\left[\left[\begin{array}{c}
\mathbf{Z}_{\mathrm{e}} \\
\mathbf{I}_{3}
\end{array}\right] \boldsymbol{\tau}+\left[\begin{array}{c}
\mathbf{f}_{\mathrm{e}} \\
-\tilde{\boldsymbol{\tau}}
\end{array}\right]\right]^{\mathrm{t}}\left[\begin{array}{cc}
\mathbf{W}_{\mathrm{f}} & \mathbf{0} \\
\mathbf{0} & \mathbf{W}_{\boldsymbol{\tau}}
\end{array}\right]\left[\left[\begin{array}{c}
\mathbf{Z}_{\mathrm{e}} \\
\mathbf{I}_{3}
\end{array}\right] \boldsymbol{\tau}+\left[\begin{array}{c}
\mathbf{f}_{\mathrm{e}} \\
-\tilde{\boldsymbol{\tau}}
\end{array}\right]\right](16)
$$

By using $\boldsymbol{\tau}$ in Eq. (14) and solving $\frac{\mathrm{d} J}{\mathrm{~d} \boldsymbol{\eta}}=\mathbf{0}$ for $\boldsymbol{\eta}$, the general solution for $\boldsymbol{\eta}$ minimizing $\mathrm{J}$ is found:

$$
\boldsymbol{\eta}=[\mathbf{R P N}]^{+}\left[-\mathbf{R Q}-\mathbf{R P A}^{+} \mathbf{b}\right]
$$

in which the following definitions have been used:

$$
\mathbf{P}=\left[\begin{array}{l}
\mathbf{Z}_{\mathrm{e}} \\
\mathbf{I}_{3}
\end{array}\right], \quad \mathbf{Q}=\left[\begin{array}{c}
\mathbf{f}_{\mathrm{e}} \\
-\tilde{\boldsymbol{\tau}}
\end{array}\right], \quad \mathbf{W}=\left[\begin{array}{cc}
\mathbf{W}_{\mathrm{f}} & \mathbf{0} \\
\mathbf{0} & \mathbf{W}_{\boldsymbol{\tau}}
\end{array}\right], \quad \mathbf{R}=\mathbf{N}^{\mathrm{t}} \mathbf{P}^{\mathrm{t}} \mathbf{W}
$$

If one wishes to maintain zero load on the primary backbone, regardless of the loads on the secondary backbones, then $\mathbf{w}_{\tau}=0$ and $\mathbf{w}_{\mathrm{f}}=\mathbf{I}_{3 \times 3}$ can be used. This is equivalent to solving Eq. (11) with Eq. (13) as a "secondary task", [17]. The general solution Eq. (17) simplifies to:

$$
\boldsymbol{\eta}=\left(\mathbf{Z}_{\mathrm{e}} \mathbf{N}\right)^{+}\left(-\mathbf{f}_{\mathrm{e}}-\mathbf{Z}_{\mathrm{e}} \mathbf{A}^{+} \mathbf{b}\right)
$$

\section{SIMULATION RESULTS}

We performed static simulations throughout a predefined workspace to compare the SLU of Fig. 1 with SLU's that use wire actuation and a single flexible primary backbone. The parameters used for these simulations are given in Table I. All symbols in this table were defined in sections III and VI, except for $\mathrm{d}_{\mathrm{o}}$ and $\mathrm{d}_{\mathrm{i}}$ that designate the outside diameters and the bores of the backbone tubes. The wrench $\mathbf{w}_{\mathrm{e}}$ was defined as the equivalent of a $1 \mathrm{~N}$ force and $1 \mathrm{Nmm}$ moment acting on the tip of the SLU.

TABLE I

THE PARAMETERS USED FOR THE SIMULATION RESULTS OF FIGURE 7

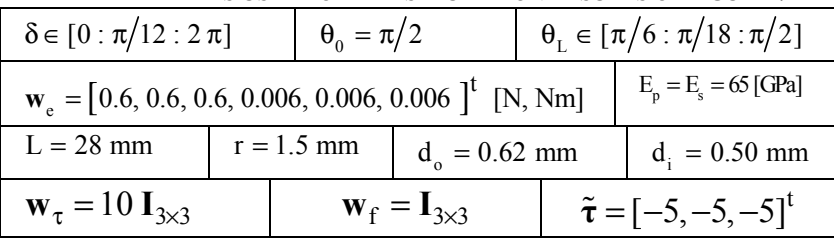

In our kinematic model, tension forces are defined negative and compression forces positive according to the definition of $\Delta \mathbf{L} \equiv\left[\Delta \mathrm{L}_{1}, \Delta \mathrm{L}_{2}, \Delta \mathrm{L}_{3}\right]^{\mathrm{t}}$. The ultimate strength of the backbones is $1070 \mathrm{MPa}$. For the combined optimization, the weights $\mathbf{w}_{\tau}, \mathbf{w}_{\mathrm{f}}$ were set such that the actuation axial stress is less than $500 \mathrm{MPa}$ throughout the SLU workspace.

Figure 7 presents the results of our static simulations. The left column shows the actuation forces and the load on the primary backbone when using a multi-backbone SLU with push-pull actuation based on the general combined optimization of Eq. (17). The right column shows the corresponding results for a wire-actuated SLU that uses a single flexible backbone. Note that the manifolds of the forces on the backbones when using a multi-backbone SLU are smoother than in the case of a wire-actuated SLU. The case of minimal effort solution ( $\boldsymbol{\eta}=\mathbf{0}$ in Eq. (14)) was also simulated and its results are shown in table II, which summarizes the results of Fig. 7. Both this table and Fig. 7 show that the compressive load on the primary backbone for the multi-backbone SLU is much smaller than in the wire actuated case. Also, for a small price of increasing the tension forces in the backbones and allowing a small compression load on the primary backbone, the maximal compression loads on all backbones are kept small (less than $36 \mathrm{~N}$ ). The maximal compression load is $59.52 \mathrm{~N}$ for the wire-actuated SLU compared to $35.32 \mathrm{~N}$ for the multibackbone SLU that uses the combined optimization. Accordingly, we believe that the multi-backbone SLU with push-pull actuation and the combined optimization actuation redundancy is more compatible for small-size snake like robots than wire-actuated SLU's.

\section{CONCLUSIONS}

This paper reported our recent work on designing a Distal Dexterity Unit (DDU) for Laryngeal minimally invasive surgery. One of the main components of this DDU is a Snake-Like Unit (SLU) that uses a novel multi-flexiblebackbone design and implements actuation redundancy. We examined the advantages of this design in terms its potential downsize scalability. We based our comparison on the premise that the reduction of compressive forces in the backbones reduces their risk of buckling and facilitates downsize scalability. We reviewed the kinematic and static modeling of this SLU and compared it to the other alternative designs implementing a single wire-actuated 
flexible backbone. The results of this paper show that the load on the central backbone can be significantly reduced in the multi-backbone design compared to designs that use wire actuation and a single flexible backbone. For these reasons we believe that the multi-backbone design is more suited for miniaturization since it allows using the push-pull actuation and actuation redundancy to prevent buckling of the backbones. Although this paper is limited to modeling and simulation, we plan to support its results by experimental validation and exact models that determine the buckling limits as a function of the geometry of the snake and its design parameters.

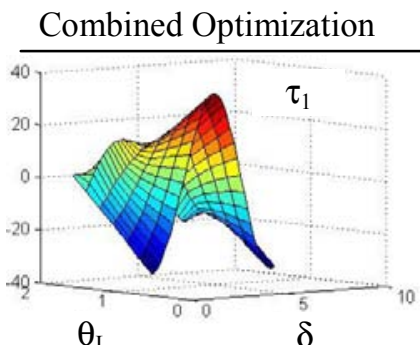

$\theta_{\mathrm{L}}$

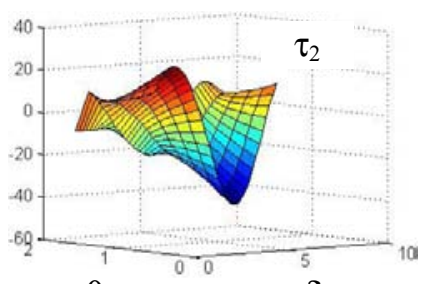

$\theta_{\mathrm{L}}$

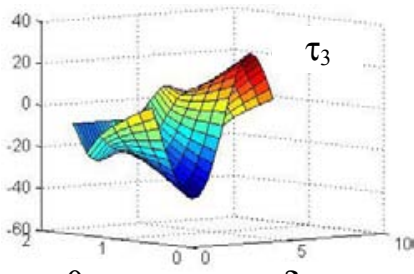

$\theta_{\mathrm{L}}$

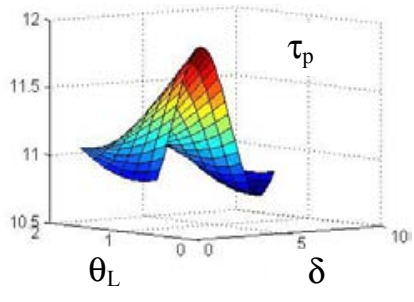

Wire Actuation

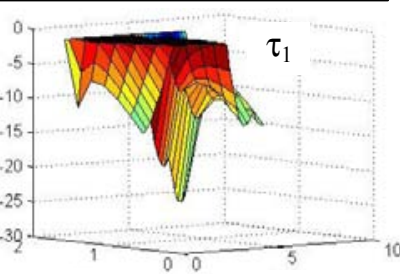

$\theta_{\mathrm{L}}$

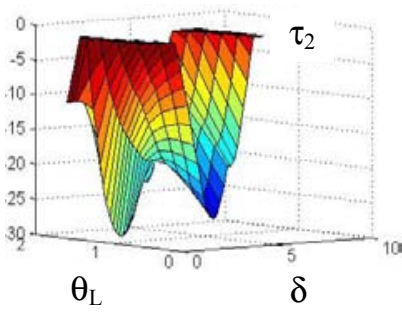

$\theta_{\mathrm{L}}$
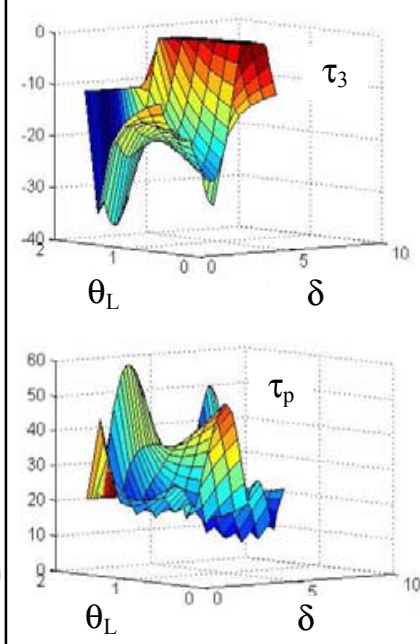

Fig. 7 Loads on secondary and primary backbones: Multi-backbone SLU using push-pull actuation and combined optimization (left). Wire actuated SLU (right). All forces are in Newton and all angles in radian.

TABLE II

MAXIMAL TENSION AND COMPRESSION FORCES ON THE BACKBONES.

\begin{tabular}{|c|c|c|c|}
\hline & Min effort & Combined optimization & Wire actuation \\
\hline$\tau_{1}$ & $-28.83,39.15$ & $-32.74,35.32$ & $-25.33,-1.00$ \\
\hline$\tau_{2}$ & $-36.34,31.11$ & $-40.22,27.24$ & $-29.83,-1.00$ \\
\hline$\tau_{3}$ & $-36.52,31.36$ & $-40.33,27.44$ & $-36.48,-1.00$ \\
\hline$\tau_{\mathrm{p}}$ & $-1.03,0.43$ & $10.74,11.87$ & $8.88,59.52$ \\
\hline $\begin{array}{l}\text { All forces are in Newton. Negative forces indicate tension. Positive } \\
\text { forces indicate compression. }\end{array}$
\end{tabular}

\section{ACKNOWLEDGEMENTS}

This work was partially funded by the National Science Foundation (NSF) under Engineering Research Center grant \#EEC9731478, NSF grant \#IIS9801684, and by the Johns Hopkins University internal funds. The extraordinary work of both Ankur Kapoor and Peter Kazanzides on designing and constructing the electronic controls and their help in the experiments are acknowledged. The author is also thankful for the guidance and support of Russell H. Taylor.

\section{REFERENCES}

[1] N. Simaan, R. Taylor, and P. Flint, "A Dexterous System for Laryngeal Surgery - Multi-Backbone Bending Snake-like Slaves for Teleoperated Dexterous Surgical Tool Manipulation," presented at IEEE International Conference on Robotics and Automation, New Orleans, pp. 351-357, 2004.

[2] N. Simaan, "High Dexterity Snake-like Robotic Slaves for Minimally Invasive Telesurgery of the Upper Airway," presented at MICCAI 2004 (7th International Conference on Medical Image Computing and Computer-Assisted Intervention), pp. 17-24, 2004.

[3] S. Hirose, Biologically Inspired Robots, Snake-Like Locomotors and Manipulators: Oxford University Press, 1993.

[4] M. Rosheim, Robot Evolution: The Development of Anthrobotics, First ed: Wiley-Interscience, 1994.

[5] G. Chirikjian and J. Burdick, "Kinematically Optimal HyperRedundant Manipulator Configurations," IEEE Transactions on Robotics and Automation, vol. 11, pp. 794-806, 1995.

[6] K. Zanganeh and J. Angeles, "The Inverse Kinematics of HyperRedundant Manipulators Using Splines," presented at IEEE International Conference on Robotics and Automation, pp. 2797-2802, 1995.

[7] I. Gravagne and I. Walker, "On the Kinematics of Remotely-Actuated Continuum Robots," presented at IEEE International Conference on Robotics and Automation, pp. 2544-2550, 2000.

[8] I. Gravagne and I. Walker, "Kinematic Transformations for RemotelyActuated Planar Continuum Robots," presented at IEEE International Conference on Robotics and Automation, pp. 19-26, 2000.

[9] C. Li and C. Rhan, "Design of Continuous Backbone, Cable-Driven Robots," ASME Journal of Mechanical Design, vol. 124, pp. 265-271, 2002.

[10]P. Dario, C. Paggetti, N. Troisfontaine, E. Papa, T. Ciucci, M. C. Carrozza, and M. Marcacci, "A Miniature Steerable End-Effector for Application in an Integrated System for Computer-Assisted Arthroscopy," presented at IEEE International Conference on Robotics and Automation, pp. 1573-1579, 1997.

[11]D. Reynaerts, J. Peirs, and H. Van Brussel, "Shape memory microactuation for a gastro-intesteinal intervention system," Sensors and Actuators, vol. 77, pp. 157-166, 1999.

[12] G. Guthart and K. Salisbury, "The Intuitive ${ }^{\mathrm{TM}}$ Telesurgery System: Overview and Application," presented at IEEE International Conference on Robotics and Automation, pp. 618-621, 2000.

[13]H. Yamashita, N. Hata, M. Hashizume, and T. Dohi, "Handheld Laprascopic Forceps manipulator Using Multi-slider Linkage Mechanisms," presented at MICCAI 2004 (7th International Conference on Medical Image Computing and Computer-Assisted Intervention), pp. 121-128, 2004.

[14]K. Ikuta, K. Yamamoto, and K. Sasaki, "Development of Remote Microsurgery Robot and New Surgical Procedure for Deep and Narrow Space," presented at IEEE International Conference on Robotics and Automation, pp. 1103-1108, 2003.

[15]E. Dombre, M. Michelin, F. Pierrot, P. Poignet, P. Bidaud, G. Morel, T. Ortmaier, D. Salle, N. Zemiti, P. Gravez, M. Karouia, and N. Bonnet, "MARGE Project: Design, Modelling, and Control of Assistive Devices for Minimally Invasive Surgery," presented at MICCAI 2004 (7th International Conference on Medical Image Computing and Computer-Assisted Intervention), pp. 1-8, 2004.

[16] M. Mason and K. Salisbury, Robot hands and the mechanics of manipulation: MIT Press, 1985.

[17] T. Yoshikawa, Foundations of Robotics Analysis and Control: MIT Press, 1990. 